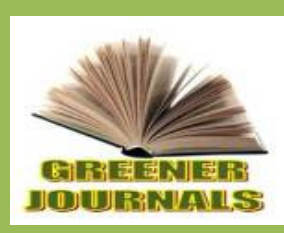

\title{
Genitourinary Fistulae Experience in a University Teaching Hospital: A South-South Nigeria Perspective
}

\author{
Inimgba N.M. ${ }^{*}$ (FWACS, FICS), John C.O.'(MBBS, FWACS), \\ Ekeke N.0.2 (FWACS, FICS)
}
${ }^{1}$ Urogynaecology/Gynaeoncology Unit, Department of Obstetrics and Gynaecology. University of Port Harcourt Teaching Hospital, PMB 6173, Port Harcourt, Rivers State, Nigeria.
${ }^{2}$ Urology unit, Department of Surgery. University of Port Harcourt Teaching Hospital, Port Harcourt, Rivers State, Nigeria.

\section{ARTICLE INFO}

Article No.: 010818003

DOI: 10.15580/GJMS.2018.1.010818003

Submitted: 08/01/2018

Accepted: $15 / 01 / 2018$

Published: 30/01/2018

${ }^{*}$ Corresponding Author

Dr. N.M. Inimgba

E-mail: nestorinimgba@yahoo. com

Phone: +2348033100433

Keywords: Genitourinary fistula, health care, university teaching hospital.

\section{ABSTRACT}

Background: Genitourinary fistula is a debilitating condition affecting women globally with high incidences in developing countries. It remains an indicator of the quality of health care delivery women are offered.

Objective: To present the experiences of genitourinary fistulas (GUF) at the University of Port Harcourt Teaching Hospital (UPTH), Rivers State.

Methods: From January 2002 to December 2012, 104 consecutive patients with genitourinary fistulas were admitted for evaluation, treatment (surgical) and thereafter followed up. Demographic and clinical data were collected. The patients were re-evaluated 3 months after surgery.

Results: Among the 67 patients treated surgically, 75\% were aged between 18 and 36 years; $46(44.2 \%)$ had vaginal repairs while $21(20.1 \%)$ had abdominal repairs. The fistulas were caused majorly by prolonged obstructed labour (72.4\%) with only 6 non obstetric causes. 37 of these patients are yet to be repaired after an initial examination under anaesthesia that was performed for every patient. These admissions do not represent or reflect the incidence of genitourinary fistulas in this centre. These characteristics provide data towards the development of an obstetric fistula prevention program in Rivers State as one presently does not exist.

Conclusion: There is an increasing incidence of GUF in this environment basically due to poor obstetric care despite different strategies proposed by different studies to the government to prevent and eradicate GUF. It also demonstrates the ability of repair or cure to restore the dignity of our women if proper training and retraining of residents and specialists, funding and making health care and facilities available to these women. 


\section{INTRODUCTION}

Genitourinary fistulae complicate obstetrics and gynaecological conditions. These complications with their profound implications, impact on the psychological, financial and physical well-being of women and their families as genitourinary fistulas remain distressing and an unresolved contributor to reproductive health morbidity among women in developing countries ${ }^{1}$.

Many factors have been identified and contributed to the persistence of this preventable problem especially in our environment.

Genitourinary fistulas are still widespread in sub-Saharan Africa, South Asia, and certain Arab States, and their consequences represent a blatant violation of fundamental human rights ${ }^{2}$. Their incidence and prevalence are unknown as in most African countries that rely mostly on hospital based data, obtaining these national data involves major challenges. Even though high maternal mortality rates remain the most important issue concerning women's health today, the problem posed by genitourinary injury in its most dreaded form, a genital fistula, is also serious $^{3}$.

Many women could be living with and suffering from the physical, psychological, and socio-economic consequences of uncontrolled urine loss due to fistula, many more than the current facility-based data suggest. This may be because the greater numbers of obstetric injuries as seen from referrals and presentation to this centre occur in the rural areas, at traditional birth attendance place or at health facilities where appropriate medical care is not readily accessible.

We share our experience in managing these cases over the past 10 years in the University of Port Harcourt Teaching Hospital.

\section{METHODS AND MATERIALS}

Data on all genitourinary fistulas seen at the hospital were obtained retrospectively from the patients' records for 2002 through 2012. This presentation summarizes and discusses the trends observed on patients admitted through the gynaecological clinic into the gynaecological ward at University of Port Harcourt Teaching Hospital, Rivers State, Nigeria. It is a 513bed hospital located at Alakahia in Obio-Akpor local government area of Rivers state South-South Nigeria. It is about fifteen kilometers from Port Harcourt city along the Port Harcourt-Warri East-West road. It is a tertiary health institution that provides all levels of health care services for Rivers, Bayelsa, Delta, Imo, Abia and Akwa-lbom states. More than 4000 deliveries are performed annually at University of Port Harcourt
Teaching Hospital, where the Caesarean delivery rate now stands at $50.3 \%{ }^{4}$.

Irrespective of the cause of the fistula, all cases except two, presented to the gynaecological clinic following referral. A complete history was taken from all women with obstetric fistulas before they underwent a complete physical examination. The history was taken with the assistance of interpreters in certain situations. Detailed information concerning the pregnancy proceeding of the fistula was also obtained; it included duration of labour, mode and place of delivery, complications, and outcome. The physical examination assessed the general health of the patient and whether neurologic (foot drop) and dermatologic lesions were present. The location, size, and number of fistulas, as well as the degree of scarring were determined with an examination under anaesthesia and dye test. When indicated, surgical treatment was offered and consent obtained. While most fistula repairs were done vaginally, some others were repaired by the abdominal route. The patients were evaluated 3 months postoperatively. They were considered cured when no leakage was seen on inspection and no incontinence was self-reported.

Regardless of the closure status of the fistulous tract, the surgical intervention was considered to have failed when the patients still was incontinent. In the absence of an internationally accepted staging system, no attempt was made to stage the fistulas at the time of examination.

\section{RESULTS}

From the 2002 to 2012 there were 104 admissions into the gynaecological ward and theatre for different genitourinary fistulas. All 104 had examinations under anaesthesia (EUA).

46 of them had vaginal repairs (44.2\%) and 21 had abdominal repairs (20.2\%). 37 are yet to be repaired following EUA, most of whom do not return for various reasons, mostly financial constraints or lost to follow up (37.57\%). Of the 104 admitted, 98 (94.2\%) were of obstetrics origin. Obstetrics origins of these fistulas were largely from prolonged obstructed labour $(74.3 \%)$, all of which were unbooked patients and referred from various traditional birth attendants' places, health centres and churches. $18.1 \%$ of the patients had prolonged labour associated with previous caesarean sections. An attempted destructive procedure (craniotomy) was associated with a rectovaginal fistula and was repaired vaginally.

Table 1 shows case distribution. This population is not the reflection of the number of patients that present to the gynaecological clinic for genitourinary fistula. 
Table 1 Cases of genitourinary fistula at University of Port Harcourt Teaching Hospital, 2002-2012

\begin{tabular}{|l|ll|}
\hline ORIGIN & NO. & $\%$ \\
\hline Prolonged Obstructed Labour & 78 & 74.3 \\
Prolonged Obstructed Labour and Caesarean & 19 & 18.1 \\
Section & & \\
Destructive operation (craniotomy) & 1 & 1.0 \\
Ruptured uterus & 1 & 1.0 \\
Total abdominal Hysterectomy & 4 & 3.8 \\
Vaginal Hysterectomy & 2 & 1.9 \\
Total & 104 & 100 \\
\hline
\end{tabular}

The youngest patient from obstetric origin was 18 years while the oldest was 45 years. The latter presented with a 4 year history of total incontinence. The average age was 23.4 years. All non-obstetrics cases were above 50 years. Three quarters of the women were below the age of 35 .

Table 2: Age distribution of patients admitted for genitourinary fistula in UPTH. 2002-2012.

\begin{tabular}{|l|l|l|}
\hline AGE & Frequency & percentage \\
\hline $18-25$ & 21 & 20.0 \\
\hline $26-35$ & 63 & 61 \\
\hline $36-45$ & 13 & 12 \\
\hline$>45$ & 7 & 7 \\
\hline
\end{tabular}

Mid vaginal fistula accounted for $62.9 \%$ of the total fistulas. Patients that were repaired with mid vaginal fistula had vaginal repairs while the majority of abdominal repairs were juxta- cervical. $5.7 \%$ had both a rectovaginal and vesicovaginal fistula. Fig 1 shows the distribution of fistula type among the population admitted.

\section{TYPE OF FISTULA}

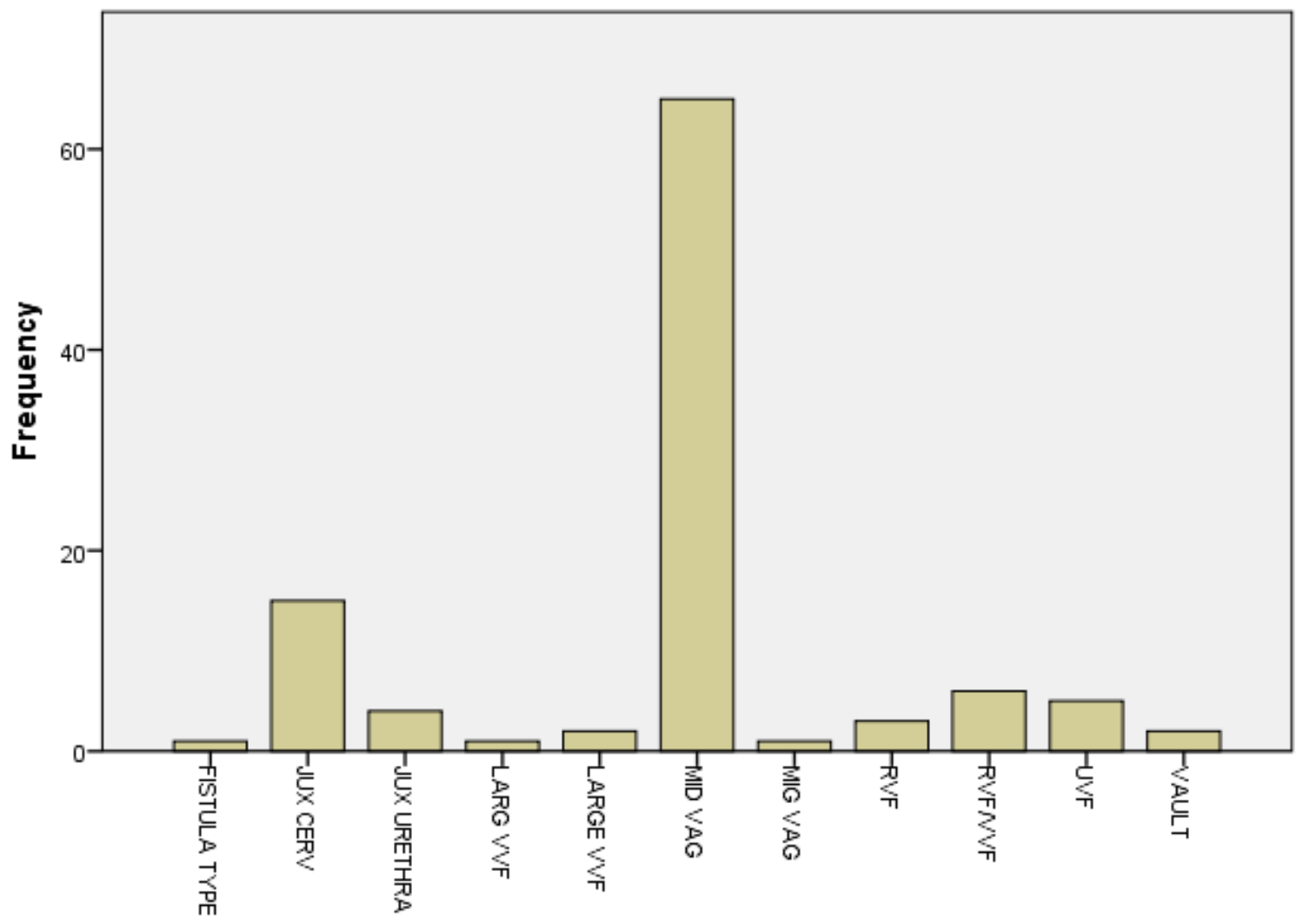

TYPE OF FISTULA

Figure 1: The distribution of fistula type 
Abdominal repairs in 3 patients required a left ureteroneocystotomy in two patients and a colostomy as a first step procedure. Both patients with left ureteroneocystotomy were complications of a caesarean section and a total abdominal hysterectomy respectively. Residual fistulas occurred in 4 patients who had vaginal repairs. These were subsequently repaired.

\section{DISSCUSSION}

Vesicovaginal fistula is a preventable disease but is prevalent among the less privileged and marginalized members of the population; the poor, young, illiterate girls and women in the remote rural areas of the world, where access to emergency obstetric care, family planning services and skilled birth attendance are unavailable and where they are available, are poorly utilized. $^{5}$ As illustrated by this study all cases of obstetric origin were unbooked women.

The effect of GUF is far reaching beyond the disease itself and thus regarded as the most dehumanizing condition to afflict women. ${ }^{6,7}$ The physical and psychosocial complications suffered by the women and relations leads to these women treated as outcasts, divorced and in many cases sex slaves, to make a living. Complications such as dysparunia, infertility, amenorrhea and gynaestresia also exist. ${ }^{8,9}$ The need for a "cure" or repair is therefore important to preserve not just the integrity of the woman but reduce the morbidities and mortalities associated with this disease. GUF is continually described as a 'preventable disease' and much is being addressed to this effect. This trend will change if the women embrace antenatal care, family planning services and effective emergency obstetric care. But the sociocultural and economic factors associated with this disease have made it difficult to prevent. Also, the constellations of the three delay model seen in most developing countries are contributory.

In Nigeria, there are contrary beliefs that GUF

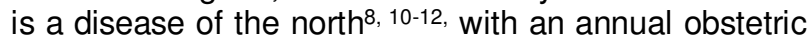
fistula incidence estimated at 2.11 per 1,000 deliveries. ${ }^{13}$ Previous studies have shown this to be wrong with GUF only more prevalent in Northern Nigeria following differences in cultural and lifestyle reasons. ${ }^{8,10-12}$ There appears to be an increase in the incidence of genitourinary fistulas in this centre. A previous study had reported 45 cases in 14 years. ${ }^{14}$ Although this study does not take into consideration the incidence but only women who were admitted and evaluated for repair. Importantly both studies show that there is great-unmet need for fistula repairs in Port Harcourt.

The youngest reported obstetric fistula in Nigeria by Kabir et al was at age $10 .{ }^{8}$ Northern studies have reported ages 13 and $17 ; ;^{15}$ while studies in south Nigeria have reported mean ages of $29.3^{11}$ years and 30.2 years $^{12}$. Previous study in Port Harcourt (UPTH) reported mean age of 26.8 year. ${ }^{14}$ This study shows the youngest patient to be 18 years and all non obstetric cases to be less than 50 years of age. This disparity in age presentation has been explained on the early marriage in northern Nigeria and overall low contraceptive prevalence rate of $10 \% .{ }^{16}$ Both factors have illustrated poor pelvic development and increase cephalopelvic disproportion experienced by teenagers. ${ }^{17}$ With $60 \%$ of the women in this study less than 35 years of age and with more primparous women, the factors of lack of contraception and poor obstetric care are major factors contributing to this condition in this study.

Obstetric injuries are the commonest cause of GUF in sub-Saharan and south Asian countries. ${ }^{18,19}$ Pressure necrosis from prolonged obstructed labour is the most common cause of vesicovaginal fistula in Nigeria and most developing countries. . $^{814,20}$ This study illustrates this finding. Other reported causes in Nigeria include uterine rupture, advanced carcinoma of the cervix, caesarean section, forceps delivery, craniotomy and Gishiri cutting. ${ }^{8,}$ 20,21 Other rare causes not common in Nigeria include rape $^{22}$ and radiation treatment ${ }^{17}$. Hysterectomy as a cause of GUF as seen in the 6 women in this study is a common finding in industrialized countries, ${ }^{17}$ however it is not a rare entity especially in unskilled hands.

A successful surgical repair of vesicovaginal fistula depends on numerous factors such as fistula size, site, surgeons' skill, surgical technique and post operative management. ${ }^{17}$ All the women had EUA as a protocol prior to repair. This is despite contrary views to one step repair advocated by other protocols and surgeons. We routinely wait for three months from time of injury before repair to aid healing, treatment of any urinary tract infections and dermatological lesions. Some studies have contrary views as long as the area is clean and healthy. ${ }^{23}$ All abdominal repairs are done with the urologist in attendance. This explains the successful abdominal repairs in 3 patients, requiring a left ureteroneocystotomy in two patients and a colostomy as a first step procedure.

The 37 women unrepaired illustrate the unfortunate burden of the Nigeria economic crisis on women with this disease that can not only pay for the repair but also that the health facilities and health practitioners in Nigeria that have the expertise to repair vesicovaginal fistula are few to meet the backlog and thousands of new cases that occur every year. ${ }^{17}$ In this study, the financial implication accounted for majority of the patient unrepaired. This financial implication includes pre-operative and post-operative investigations, radiological assessment individualized for each patient, surgical and hospital in ward fees. In some cases the patients were lost to follow up following delays and backlog encountered.

\section{CONCLUSION}

This study concludes that there is an increasing incidence of GUF in this environment basically due to poor obstetric care despite different strategies proposed by different studies to the government to prevent and eradicate GUF. It also demonstrates the ability of repair or cure to restore the dignity of our 
women if proper training and retraining of residents and specialists, funding and making health care and facilities available to these women.

Lastly there must be need to get the true incidence and prevalence with a community based study.

\section{REFERENCES}

1. Waaldijk K, Armiya'u YD. The obstetric fistula: a major health problem still unresolved. Int Urogynecol J 1993; 4:126-8.

2. Danso KA, Martey JO, Wall LL, Elkins TE. The epidemiology of genitourinary fistulae in Kumasi, Ghana. Int Urogynecol J 1996; 7:117-20.

3. Danso KA, Opare-Addo HS, Turpin CA. Obstetric fistula admissions at Komfo Anokye Teaching Hospital, Kumasi, Ghana. Int Journal of Gynecology and Obstetrics (2007) 99, S69-S70.

4. Annual Report. Department of Obstetrics and Gynaecology, University of Port Harourt Teaching Hospital, River State. Nigeria. 2013. Unpublished.

5. Lewis G, de Bernis L. Obstetric fistula: Guiding principles for clinical management and programme development. Integrated management of pregnancy and childbirth. WHO Press. 2006: 3-6.

6. Muleta M. Obstetric fistula in developing countries: A review article. J Obstet Gynaecol Can. 2006; 28: 962-966.

7. Ijaiya MA, Aboyeji AP, ljaiya ZBB. Epidemiology of Vesico-Vaginal Fistula The University of Ilorin Teaching Hospital, Ilorin, Nigeria. Trop J Obstet Gynaecol. 2002; 19: 101-103.

8. Kabir M, Iliyasu Z, Abubakar IS, UmarUI. Medicosocial problems of patients with vesicovaginal fistula in Murtala Mohammed Specialist Hospital, Kano. Annals of African Medicine. 2004; 2:54-57.

9. MA ljaiya, AG Rahman, AP Aboyeji, AWO Olatinwo, SA Esuga, OK Ogah, HO Raji, IO Adebara, AO Akintobi, AS Adeniran, AAA Adewole. Vesicovaginal fistula: a review of the
Nigerian experience. W Afr J Med. Vol. 29( 5); 2010: 293-298.

10. Orji EO, Adeloju OP, Orji VO. Correlation and impact of obstetric fistula on motherhood. Journal of Chinese Clinical Medicine. 2007; 2: 448-454.

11. Mela GS, Massa AA, Yahaya UR, Bukar M, Kizaya DD and El-Nafaty AU. Risk factors for obstetric fistula in north-eastern Nigeria. Journal of Obstetrics and Gynaecology. 2007; 27: 819-823.

12. Audu BM, Kullima AA, Bako B. Epidemiology of vesico-vaginal fistula: No longer a calamity of teenagers. Journal of Obstetrics and Gynaecology. 2008; 28: 432-433.

13. Tsui $A O$, Creanga $A A$, Ahmed $S$. The role of delayed childbearing in the prevention of obstetric fistulas. International Journal of Gynaecology \& Obstetrics. 2007: S98-S107.

14. Inimgba NM, Okpani AOU, John CT. VesicoVaginal Fistulae in Port-Harcourt, Nigeria. Trop J Obstet Gynaecol. 1999; 16: 49-51.

15. Ibrahim T, Sadiq AU, Daniel SO. Characteristics of VVF patients as seen at the specialist hospital Sokoto, Nigeria. West Afr J Med. 2000; 19: 59-63.

16. WHO. Reproductive health indicators data base; July 2006. Monitoring And Evaluation. http://www.whoint/ reproductive_indicators/country data 6/7/2008.

17. Roy KK, Malhotra N, Kumar S, Seth A and Nayar B. Genitourinary fistula- An Experience from a Tertiary Care Hospital. JK Science - Archive. 2006; 3: 144-147.

18. Wall LL, Karshima AK, Kirschner C, Arrowsmith SD. The obstetric vesicovaginal fistula: Characteristics of 899 patents from Jos, Nigeria. American Journal of Obstetrics and Gynaecology. 2004; 190: 1011-1019.

19. Muleta M, Williams G. Postcoital injuries treated at the Addis Ababa fistula hospital, 1991-1997. Lancet.1999; 354: 2051-20512.

20. B Hancock. M Collie. Vesico-vaginal fistula surgery in Uganda. East and Central African Journal of Surgery. 2004; 9( 2): 32-37. 